\title{
Optimization of Gantry Cranes' Operation Path for Transshipment Based on Improved TSP
}

\author{
Qi Zhang $\mathbb{D}^{D}$, Hongjin Dong $\mathbb{D}^{D}$, Mingjun Ling $\mathbb{D}^{\mathbb{D}}$, Leyi Duan, and Yuguang Wei \\ School of Traffic and Transportation, Beijing Jiaotong University, Beijing 100044, China \\ Correspondence should be addressed to Hongjin Dong; 16120796@bjtu.edu.cn
}

Received 26 March 2019; Revised 10 July 2019; Accepted 14 September 2019; Published 12 January 2020

Academic Editor: Eneko Osaba

Copyright (c) 2020 Qi Zhang et al. This is an open access article distributed under the Creative Commons Attribution License, which permits unrestricted use, distribution, and reproduction in any medium, provided the original work is properly cited.

\begin{abstract}
In order to improve the transshipment efficiency of transit containers in the port or the port-type railway network container freight station (PRNCS) with the condition that each transit container matches a railway flat-car, this paper studied the optimization of operation path of the rail mounted gantry crane (RMG) in the loading and unloading track for containers transshipped directly from highway to railway. Based on the basic model of TSP, the paper constructed the optimization model for the operation path of RMG, and designed the Ant Colony Algorithm (ACA) to solve it, and then obtained the operation scheme of RMG having the highest efficiency. Finally, the validity and correctness of the model and algorithm were verified by a case.
\end{abstract}

\section{Introduction}

As the key node of transportation network for international containers, PRNCS is not only the hub for distribution of containers, but also the junction of various transportation modes for the multimodal transport of containers. Its operational efficiency not only affects the benefit and service level of the station itself, but also plays a key role in the efficient operation for the entire transportation network. At present, the main factor which restricts the transshipment efficiency of containers is that the time of "Second Customs Declaration" is too long. After all containers are loaded on a train, the manifest should be submitted to the customs. And the train could depart from the station, only when the manifest is approved by the customs. That is, the fully loaded train needs waiting for a long time in the station before departure, which seriously restricts the transportation efficiency of containers. Based on this, in the actual operation of some station, the dispatcher of PRNCS will make the "pre-manifest", after the "First Customs Declaration" is completed. The pre-manifest determines the matching relationship of containers and flat-cars in advance, that is, each transit container arriving by sea matches a railway flat-car. And then, organizing the loading operation of containers according to the matching relationship of containers and flat-car determined by the pre-manifest. At the same time, submitting the pre-manifest to the customs for the "Second
Customs Declaration". Thereby, the loading operation and the "Second Customs Declaration" are performed in parallel, which can shorten the waiting time of containers in the station. Taken the directly transshipment of containers from highway to railway as the research background, this paper studies the optimization of transshipment operation for the RMG, under the condition that each container matches a railway flat-car. This paper constructed the optimization model based on the TSP theory, and then obtained the optimal operation scheme of RMG.

In recent years, domestic and foreign scholars have conducted a lot of research on the optimization of gantry crane operations. Zhu [1] studied the task assignment between multiple loading and unloading equipment (gantry cranes and front carriers), according to the punctual and late running of trains. $\mathrm{Xu}$ [2] studied the optimal assignment of gantry cranes' task of loading and relocating the container. Briskorn [3] treats the crane scheduling in a container port where two cooperative gantry cranes (denoted as twin cranes) jointly store import containers arriving from the seaside in a storage yard. Jaehn [4] consider the problem of scheduling two identical rail mounted gantry cranes (twin cranes) working within a single storage area (block) at a seaport. And the cranes cannot pass each other. The focus is minimizing the dwell times of vessels at the berth. Chen [5] classified the task of gantry cranes into stage tasks and subtasks within the phase, and constructing the optimization 
model, in order to optimize the gantry cranes' operation path among the storage blocks. Li [6] consider single-crane scheduling at rail transshipment yards, in which gantry cranes move containers between trains, trucks and a storage area. And the objective is minimizing the train dwell time in the yard. Heshmatia [7] studied the Crane-operated Warehouse Scheduling Problem (CWSP), concerns the location assignment of input products and the scheduling of cranes for product movement in such warehouses. Al-Dhaheri [8] studied the quay cranes scheduling problem, with the objective minimizing vessel handling time while considering the entire container handling process involving both seaside operations and container transfer operations, taking place between the quay and the stacking yard. Diabat [9] studied the optimal unloading scheme of Quay Cranes, through the way balancing the workload of each Quay Crane. Kress [10] consider a scheduling problem for two gantry cranes moving on the same rails at a single storage block. And the objective considered in this article is to minimize the makespan of seaside container processing while considering non-crossing constraints among cranes. Taking the receiving and delivery operations of outside trucks as the research object, Böck [11] proposes different methods for generating a trajectory for the load based on a geometric path connecting the loading and unloading position. And the overall goal is to traverse the geometric path as fast as possible under the consideration of constraints for the gantry crane system.

TSP is widely researched and used in the transportation field. Benavent [12] approaches a pickup-and-delivery single-vehicle routing problem where there is susceptibility to uncertainty in customer requests. Taking the route choosing of container liner as the research object, Miranda [13] introduces and studies the bi-objective insular traveling salesman problem, where a set of rural islands must be served using a single barge following a single route. And two objective functions are aimed to be minimized: maritime and ground transportation costs. $\mathrm{Li}$ [14] studied the cargo distribution problem of the logistics center, constructed the optimization model with the shortest route and minimized time base on the theory of TSP.

In summary, researches on scheduling optimization of the gantry crane at present are mainly for the container yard of ports. While for the railway network container freight station, the research mainly focuses on two aspects, task allocation among loading and unloading equipment, relocating containers. The application of TSP theory in transportation field mainly focuses on route selection of carrying tools.

At present, there are no scholars have used the TSP theory to solve the problem of scheduling optimization of gantry cranes, under the condition that each container matches a railway flat-car, in the loading-unloading track.

Therefore, this paper constructed an improved TSP model, and uses it to study scheduling optimization of gantry cranes, for containers transshipping from highway to railway, under the condition that each container matches a railway flat-car.

\section{Problem Description}

The Carrier organizes container trucks coming to the loading-unloading zone, according to the arrival time of the container train. And then, these containers on trucks are loaded onto the flat-car directly by gantry cranes. However, due to the noncomplete sharing of transportation information, and the randomness of arrival for container trucks, the container truck cannot accurately align with the flat-car according to the matching relationship of containers and flat-cars.

As shown in Figure 1, the loading-unloading track is divided into two areas, and each area has only one gantry crane. In the figure, $r_{i}$ is the containers arriving at by railway, or RC for short; $s_{i}$ is the container arriving at by highway, or HC for short. Arrows in Figure 1 represent the matching relationship of containers and flat-cars determined by the pre-manifest. Therefore, in order to complete the transshipment task of containers by the shortest operation time and minimize the number of containers that were unloaded to the ground as well, it is necessary to optimize the operation path of gantry cranes. The operation process of gantry cranes for the directly transshipment operation of containers from highway to railway, under the condition of matching relationship of containers and flat-cars, is as follows.

Step 1 . The gantry crane selects a HC randomly $\left(s_{i}\right.$, $i=1,2, \ldots, m)$, and then unloads it to the ground $\left(s_{i}^{\prime}\right)$. As a result, a container vacancy will be created on the container trucks, denoted as SE.

Step 2. The gantry crane selects a RC randomly $\left(r_{k}\right.$, $k=1,2, \ldots, m)$, and then unloads it to $S E$. As a result, a container vacancy will be created on the railway flat-cars, denoted as $R E$.

Step 3. The gantry crane selects the HC matched with $R E$, denoted as $s_{R E}$, and then transfers it to $R E$. Whether all containers have been operated? If yes, the operation of the gantry crane ends; otherwise, $s_{R E}$ is $s_{i}^{\prime}$ ? If yes, go to Step 1; otherwise, a $S E$ will be generated, and go to Step 2.

The operation process of gantry cranes is shown in Figure 2.

\section{GC-TSP}

3.1. Traveling Salesperson Problem. Traveling Salesperson Problem (TSP) is a famous problem in the operational research. In graph theory, TSP appears as a closed loop that traverses all nodes in the network graph. For the network graph $G(V, E), V$ is the set of nodes, $E$ is the set of arcs, $d_{i j}$ is the length of various arcs. Then the classic mathematical programming model of TSP is as follows.

$$
\begin{aligned}
& \min Z=\sum_{i=1}^{n} \sum_{j=1}^{n} d_{i j} x_{i j}, \\
& \sum_{j=1}^{n} x_{i j}=1, \quad i \in V, \\
& \sum_{i=1}^{n} x_{i j}=1, \quad j \in V .
\end{aligned}
$$




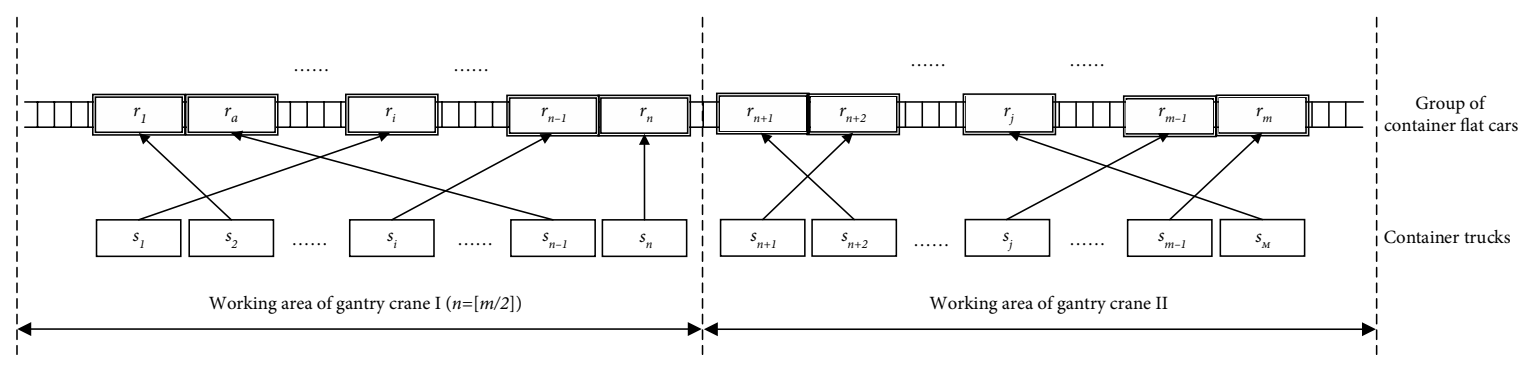

FIgURE 1: The position map of container trucks and flat cars on the loading and unloading line.

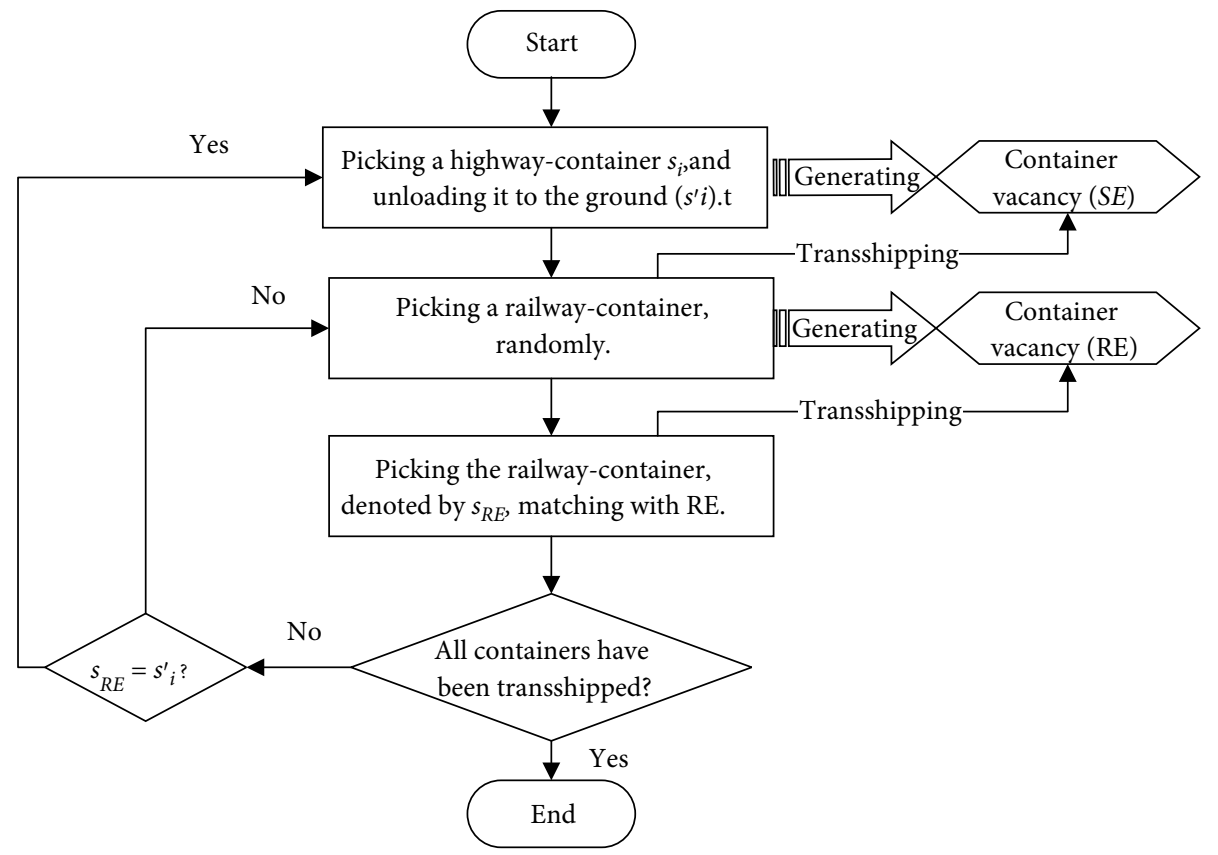

FIgURE 2: The flow chart for gantry cranes' operation.

$$
x_{i j}=\{0,1\},
$$

where, $x_{i j}$ is the $0-1$ decision variable, and $x_{i j}=1$ means that the $\operatorname{arc}(i, j)$ is included in the TSP loop, and vice versa, not in the TSP loop. The Equation (2) means that there is one and only one arc starting from each node in the network graph. Equation (3) indicates that there is one and only one arc reaches each node. That is, the TSP loop pass through and pass through each node in the network graph only once.

3.2. GC-TSP. Under the condition that each transit container (HC) matches a railway flat-car, the efficiency of transshipment is reflected by the following two aspects, the total time of transshipment and the ratio of directly transshipment between highway and railway for containers. According to the basic theory of TSP, this paper transforms the optimization problem of operation path for RMG into an improved Traveling Salesperson Problem, called GC-TSP (Gantry Crane-TSP). Designing the network diagram of operation path for gantry cranes, as shown in Figure 3. Find a shortest path of gantry cranes in the network diagram, with the objective that the total time of transshipment and the number of containers unloaded to the ground both are minimum, and that is the optimal operation plan of gantry cranes.

3.2.1. Network Diagram for Gantry Cranes. Taking a certain area of the loading and unloading line as an example, constructing the network diagram for the path of gantry cranes, as shown in Figure 3. The network diagram consists of nodes and arcs, and the $r$ type node denotes RCs, and the $s$ type node denotes HCs, and the $s^{\prime}$ type node denotes the container unloaded to the ground, and arcs in the figure denote the process of transshipment operation for gantry cranes.

According to the nature of container transshipment, the operation of gantry cranes can be divided into the following three categories: (1) the gantry crane transfers HCs to the railway flat-car matched with it; (2) the gantry crane transfer RCs to any empty container truck; (3) the gantry crane randomly selects a $\mathrm{HC}$ and unloads it to the ground. Correspondingly, arcs in the figure can be divided into following three categories. 


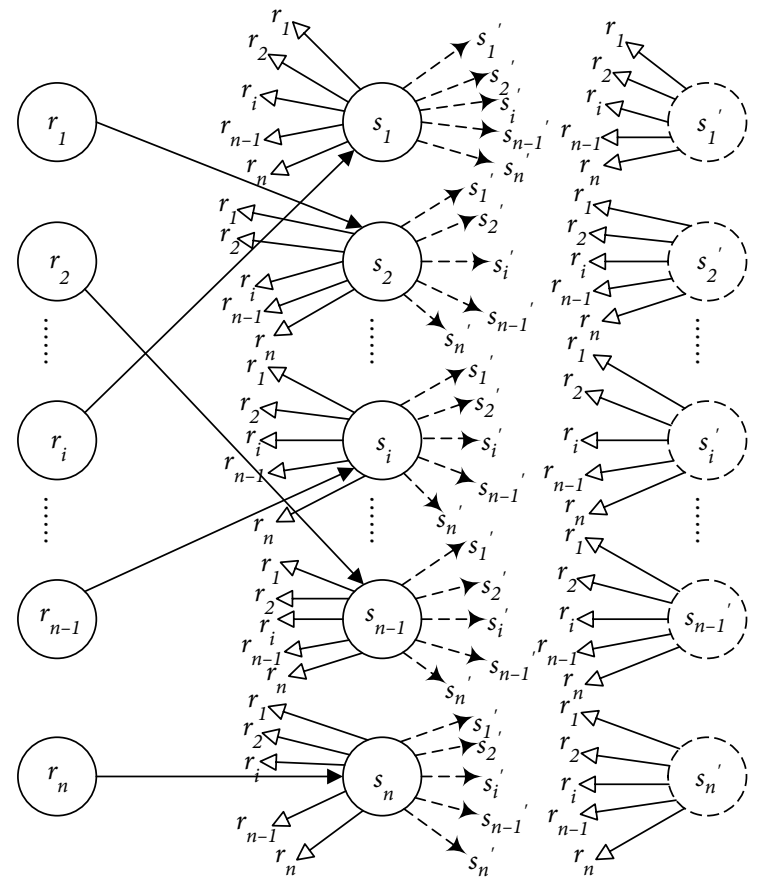

FIGURE 3: The network diagram for the path of gantry cranes.

(1) Specified Arcs. The gantry crane transfer HCs to the railway flat-car. Because that the pre-manifest has determined the matching relationship between HCs and flat-cars, a HC can only be transferred to the railway flat-car matched with it by the gantry crane. As shown in Figure 3, the gantry crane can only reach the node $s_{2}$ from node $r_{1}$, indicating that the container $s_{2}(\mathrm{HC}) \mathrm{can}$ only be transferred to the flat-car locating at $r_{1}$.

(2) Random Arcs. The gantry crane transfer HCs to any empty container truck. It should be emphasized that any container truck can be used by a RC as long as it is empty. In the Figure 3, which appears as that the $s$ type node can reach all of $r$ type nodes. Similarly, the $s^{\prime}$ type node can reach all of $r$ type nodes as well in the network diagram.

(3) Transfer Arcs. According to the matching relationship of transit containers and railway flat-cars determined by the pre-manifest, if the HC selected by the gantry crane is the container that has been unloaded to the ground, after the transshipment operation is completed, the gantry crane needs to move to the position of any un-transshipped $\mathrm{HC}$, and unloads this container to the ground for generating a new container vacancy. The process of selecting the un-transshipped HC is the transfer arc in the network diagram.

3.2.2. Dynamic Nature of GC-TSP. Figure 4 is an operation path of the gantry crane, if adding, from the end node $s_{i}$ to the start node $s_{1}^{\prime}$, a virtual arc whose operation time is zero, and deleting all of unvisited virtual nodes, therefore, converting the problem of optimizing the operation path of gantry cranes into the GC-TSP, as shown in Figure 5. Note that if a virtual node is not reached, it means that the corresponding container of the node was not unloaded to the ground.

Compared with TSP, GC-TSP has the following characteristics [15]:

(1) In the network diagram, there is not necessarily an arc between any two nodes. The matching relationship of transit containers and flat-cars determined by the pre-manifest makes a HC can only be loaded to the railway flat-car matched with it. In the network diagram, it appears as that the $r$ type node can only reach the specified $s$ type node matched with it. As shown in Figure 3, the node $r_{1}$ can only reach the node $s_{2}$. In addition, the transshipment operation cannot occur between any two RCs, which appears as that there is no arcs between any two $r$ type nodes in the network diagram. Similarly, there is no arcs between any two $s$ type nodes and $s^{\prime}$ type nodes as well.

(2) The number of nodes in the network diagram is uncertain, which changes dynamically with the advancement of the optimization process. The uncertainty of the number of containers unloaded to the ground leads to the uncertainty of the number of nodes in the network diagram.

(3) The time of arcs in the network diagram is uncertain, which changes dynamically with the advancement of the optimization process. Taking the $\operatorname{arc}\left(r_{1}, s_{2}\right)$ in the Figure 4 as an example, the operation process of the arc is that the gantry crane move to $s_{2}$, picking up container $s_{2}$, and then move to $r_{1}$, and then unload container $s_{2}$ to the railway flat-car $r_{1}$. Therefore, the end point of transshipment operation of $\operatorname{arc}\left(r_{1}, s_{2}\right)$ is $r_{1}$, and then, the start point of next $\operatorname{arc}\left(s_{2}, r_{2}\right)$ in the loop is $r_{1}$. Therefore, the time of arcs in the network diagram is related with the working path of the gantry crane. And the dynamic nature of working path leads to the dynamicity of time for each arc.

Based on the dynamic nature of the number of nodes and the time of arcs, the GC-TSP can be called "Dynamic TSP".

\section{Model Construction}

\subsection{Assumption}

(1) There is only one gantry crane in each working area.

(2) According to China Railway Express, all of containers in this paper are 40 feet.

(3) Each railway flat-car and container truck load with only one 40 feet container.

(4) The cart running gear and hoisting mechanism of the gantry crane cannot move at the same time.

(5) The operating speed of gantry cranes is constant.

(6) Only the HC can be unloaded to the ground by gantry cranes. 


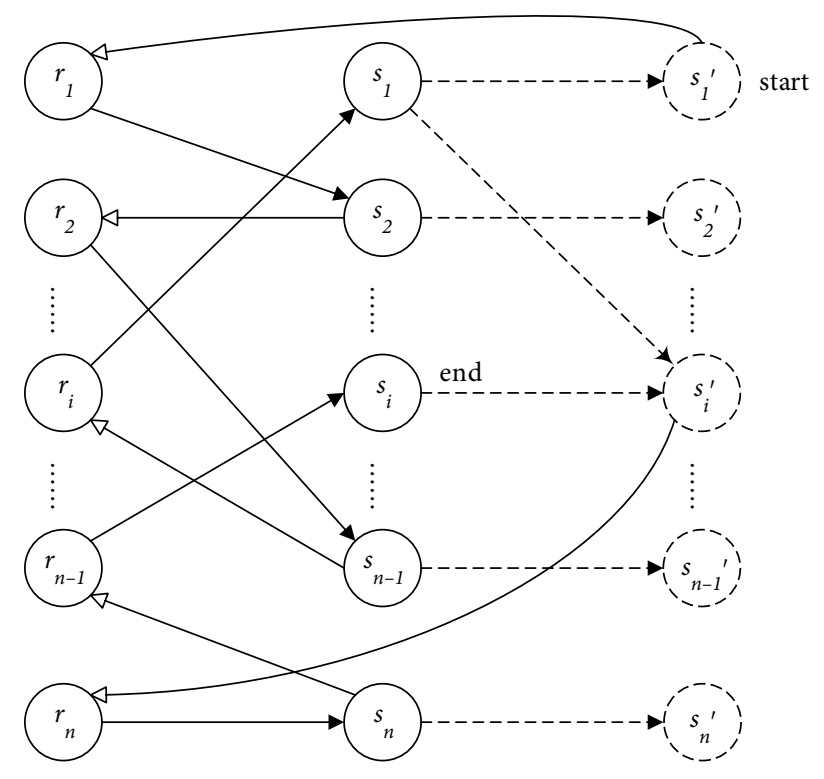

FIGURE 4: The operation path of gantry crane.

\subsection{Parameters and Variables}

\section{(1) Basic Paramters}

$t_{\mathrm{e}}$ - the operation time of transshipping one container by the hoisting mechanism, Unit: min.

$v_{\mathrm{b}}$-the speed of the cart running gear, Unit: $\mathrm{m} / \mathrm{min}$.

$l$-the length occupied by one container in the loading and unloading line, Unit: $m$.

$m$-the number of railway flat-cars.

$\omega$-the number of gantry cranes.

\section{(2) Decision Variables}

$x_{s(i) r(j)}$-if the gantry crane transships the container $r_{j}$ to the truck located at $s_{i}$, it equals 1 ; otherwise, 0 . (Random Arcs.)

$x_{s^{\prime}(i) r(j)}$-if the gantry crane transships the container $r_{j}$ to the truck located at $s_{i}^{\prime}$, it equals 1 ; otherwise, 0 . (Random Arcs.)

$y_{r(j) s(i)}$-if the gantry crane transships the container $s_{i}$ to the truck located at $r_{j}$, it equals 1 ; otherwise, 0 . (Specified Arcs.)

$z_{s(i) s^{\prime}(i)}$-if the gantry crane unloads the containers $s_{i}$ to the ground, it equals 1 , otherwise, 0 .

$z_{s(i) s^{\prime}(j)}^{\prime}$-in order to generated a new container vacancy, if the gantry crane selects the container $s_{j}$, it equals 1; otherwise, 0. (Transfer Arcs.)

$\zeta$-the variable for indexing a gantry crane,

$\zeta=1,2, \ldots, \omega$.

$T^{\zeta}$-the total operation time of the gantry crane

$\zeta, \zeta=1,2, \ldots, \omega$.

$E_{T N}^{\zeta}$-the operation efficiency of gantry crane $\zeta, \zeta=1,2, \ldots, \omega$.

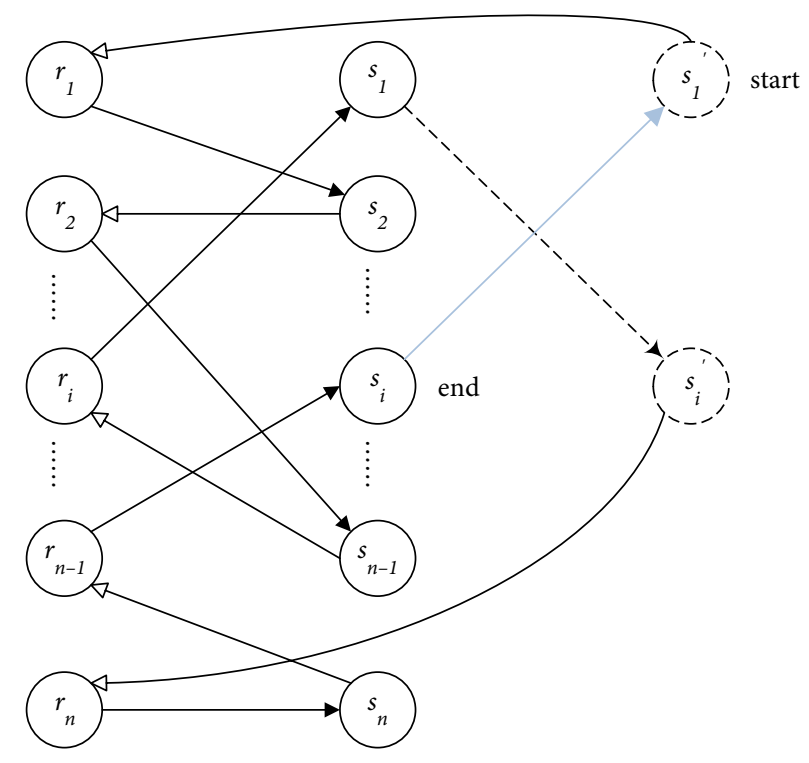

FIGURE 5: The gantry crane-TSP loop.

$T^{* \zeta}$-taking the shortest operation time as the single objective, the optimal time of gantry crane $\zeta, \zeta=1,2, \ldots, \omega$.

$N^{* \zeta}$-taking the minimum number of containers unloaded to the ground as the single objective, the optimal value of gantry crane $\zeta, \zeta=1,2, \ldots, \omega$.

$E_{T N}^{* \zeta}$ - the optimal operation efficiency of gantry crane $\zeta, \zeta=1,2, \ldots, \omega$.

\section{(3) Collection Variables}

$R=\left\{r_{1}, r_{2}, \ldots, r_{m}\right\}$-the collection of HCs.

$S=\left\{s_{1}, s_{2}, \ldots, s_{m}\right\}$-the collection of RCs.

$S^{\prime}=\left\{s_{1}^{\prime}, s_{2}^{\prime}, \ldots, s_{m}^{\prime}\right\}$-the collection of containers that were unloaded to the ground.

$G=\left(g_{i j}\right)_{m \times 2}=\left(G_{r}, G_{s}\right)$-the matching relationship of containers and flat-cars.

$Y_{R S}=\left\{y_{r(j) s(i)}=y_{G r(k) G s(k)}=1\right\}$ - the collection of variables corresponding to the specified arcs, $k=1,2, \ldots, m$.

$P=\{1,2, \ldots, m\}$-the collection of location where the gantry crane moves, and numbering sequentially from one end of the loading and unloading line. For example, the serial number of the container $r_{i}$ and $s_{i}$ is $i, i=1,2, \ldots, m$.

$Q_{\zeta}$-the collection of location of working area $\zeta$ in the loading and unloading line, $\zeta=1,2, \ldots, \omega$.

$N Q_{\zeta}$-the number of elements in $Q_{\zeta}, \zeta=1,2, \ldots, \omega$. list $\zeta_{1}^{\zeta}=\left\{s^{\prime}(i), r(j), s(k), \ldots, s^{\prime}(i)\right\}$ - the sequence table of containers that were operated by the gantry crane $\zeta, \zeta=1,2, \ldots, \omega$.

list ${ }_{2}^{\zeta}=\{i, j, k, \ldots, i\}$-the sequence table of location for the transshipment operation of the gantry crane $\zeta, \zeta=1,2, \ldots, \omega$.

$n_{\zeta}$-the number of elements in $l i s t_{1}^{\zeta}$. 
$N I S^{\zeta}$ - the noninferior solution for the optimal operation of the gantry crane $\zeta$, and $N I S^{\zeta}=\left\{\min T^{\zeta}, \min N^{\zeta}\right\}$, $\zeta=1,2, \ldots, \omega$.

\section{(4) Location Variables}

$k_{s(i) r(j)}$-the order of the starting point $s_{i}$ for the arc

$k_{s^{\prime}(i) r(j)}$-the order of the starting point $s_{i}^{\prime}$ for the arc $\left(s_{i}^{\prime}, r_{j}\right)$ in $l i s t_{1}^{\zeta}$.

$k_{r(j) s(i)}$-the order of the starting point $r_{j}$ for the arc $\left(r_{j}, s_{i}\right)$ in $l i s t_{1}^{\zeta}$.

$k_{s(j) s^{\prime}(j)}$-the order of the starting point $s_{i}$ for the arc $\left(r_{j}, s_{i}\right)$ in $l i s t_{1}^{\zeta}$.

(5) Time Parameters

$t_{s(i) r(j)}$-the operation time of the gantry crane for the $\operatorname{arc}\left(s_{i}, r_{j}\right), i, j=1,2, \ldots, m$.

$t_{s^{\prime}(i) r(j)}$-the operation time of the gantry crane for the $\operatorname{arc}\left(s_{i}^{\prime}, r_{j}\right), i, j=1,2, \ldots, m$. $\left(s_{i}, r_{j}\right)$ in $l i s t_{1}^{\zeta}$.

$t_{r(j) s(i)}$ - the operation time of the gantry crane for the $\operatorname{arc}\left(r_{j}, s_{i}\right), i, j=1,2, \ldots, m$.

$t_{s(i) s^{\prime}(j)}$-the operation time of the gantry crane for the $\operatorname{arc}\left(s_{i}, s_{j}^{\prime}\right), i, j=1,2, \ldots, m$, and $i \neq j$.

$t_{\text {end }}^{\zeta}$ - the operation time of the virtual arc in working path of the gantry crane.

\subsection{GC-TSP Model}

$$
\begin{aligned}
\min T^{\zeta}= & \min \left(\sum_{i, j \in Q^{\zeta}} t_{s^{\prime}(i) r(j)} \times x_{s^{\prime}(i) r(j)}+\sum_{i, j \in Q^{\zeta}} t_{s(i) r(j)} \times x_{s(i) r(j)}\right. \\
& +\sum_{y_{r(j) s(i)} \in Y_{R S}} t_{r(j) s(i)} \times y_{r(j) s(i)}+\sum_{i, j \in Q^{\zeta}} t_{s(i) s^{\prime}(j)} \times z_{s(i) s^{\prime}(j)}^{\prime} \\
& \left.+\sum_{i \in Q^{\zeta}} t_{e} \times z_{s(i) s^{\prime}(i)}\right), \\
& \min _{s^{\prime}(i) r(j)}=2 \times \frac{\min \left(n_{\zeta}-2 N Q_{\zeta}-1\right),}{v_{b}}+l i s t_{2}^{\zeta}\left(k_{s^{\prime}(i) r(j)}+1\right)-l\left(k_{s^{\prime}(i) r(j)}\right) \mid \times l
\end{aligned}
$$

$t_{s(i) s^{\prime}(j)}=\frac{\left|l i s t_{2}^{\zeta}\left(k_{s(i) s^{\prime}(j)}+1\right)-l i s t_{2}^{\zeta}\left(k_{s(i) s^{\prime}(j)}-1\right)\right|}{v_{b}} \times l$,

$$
t_{\text {end }}^{\zeta}=0, \zeta=1,2, \cdots, \omega
$$

$$
\sum_{s_{i}^{\prime} \in S^{\prime}} x_{s^{\prime}(i) r(j)}+\sum_{s_{i} \in S} x_{s(i) r(j)}=1, \quad j=1,2, \cdots, m,
$$

$$
\sum_{s_{i} \in S} y_{r(j) s(i)}=1, \quad j=1,2, \cdots, m
$$

$$
\sum_{r_{j} \in R} x_{s(i) r(j)}+\sum_{s_{j}^{\prime} \in S^{\prime}} z_{s(i) s^{\prime}(j)}^{\prime}=1, \quad i=1,2, \cdots, m
$$

$$
\sum_{r_{j} \in R} x_{s^{\prime}(i) r(j)}=z_{s(i) s^{\prime}(i)}, \quad i=1,2, \cdots, m,
$$

$$
\sum_{r_{j} \in R} y_{r(j)_{s(i)}}=1, \quad i=1,2, \cdots, m
$$

$$
\begin{gathered}
\sum_{s_{i} \in S} z_{s(i) s^{\prime}(j)}^{\prime}=z_{s(j) s^{\prime}(j)}, \quad j=1,2, \cdots, m, \\
\operatorname{list}_{1}^{\zeta}(1)=\operatorname{list}_{1}\left(n_{\zeta}\right)
\end{gathered}
$$

$$
x_{s(i) r(j)}, x_{s^{\prime}(i) r(j)}, y_{r(j) s(i)}, z_{s(i) s^{\prime}(j)}, z_{s(i) s^{\prime}(j)}^{\prime}=\{0,1\} .
$$

The formula (5) means that the total operation time of the gantry crane is minimum. The formula (6) means that the number of containers that were unloaded to the ground is minimum. The formulas (7) and (8) are the operation time of random arcs. The formula (9) is the operation time of specified arcs. The formula (10) is the operation time of transfer arcs. The formula (11) means that only one arc reaches the node $r_{j}$. The formula (12) means that only one arc starts from the node $r_{j}$. The formula (13) means that only one arc starts from the node $s_{i}$. The formula (14) means that only one arc will start from the node $s_{i}^{\prime}$ if the containers $s_{i}$ is unloaded to the ground, otherwise, there will be no arcs starting from the node $s_{i}^{\prime}$. The formula (15) means that only one arc reaches the node $s_{i}$. The formula (16) means that only one arc will reach the node $s_{j}^{\prime}$ if the containers is unloaded to the ground, otherwise, there will be no arcs reaching the node $s_{j}^{\prime}$. The formula (17) means that the gantry crane will return to the starting point after the transshipment task is completed. The formula (18) means that the working time of the virtual arc from the end point to the starting point is 0 . The formula (19) is the $0-1$ constraint on variables. 


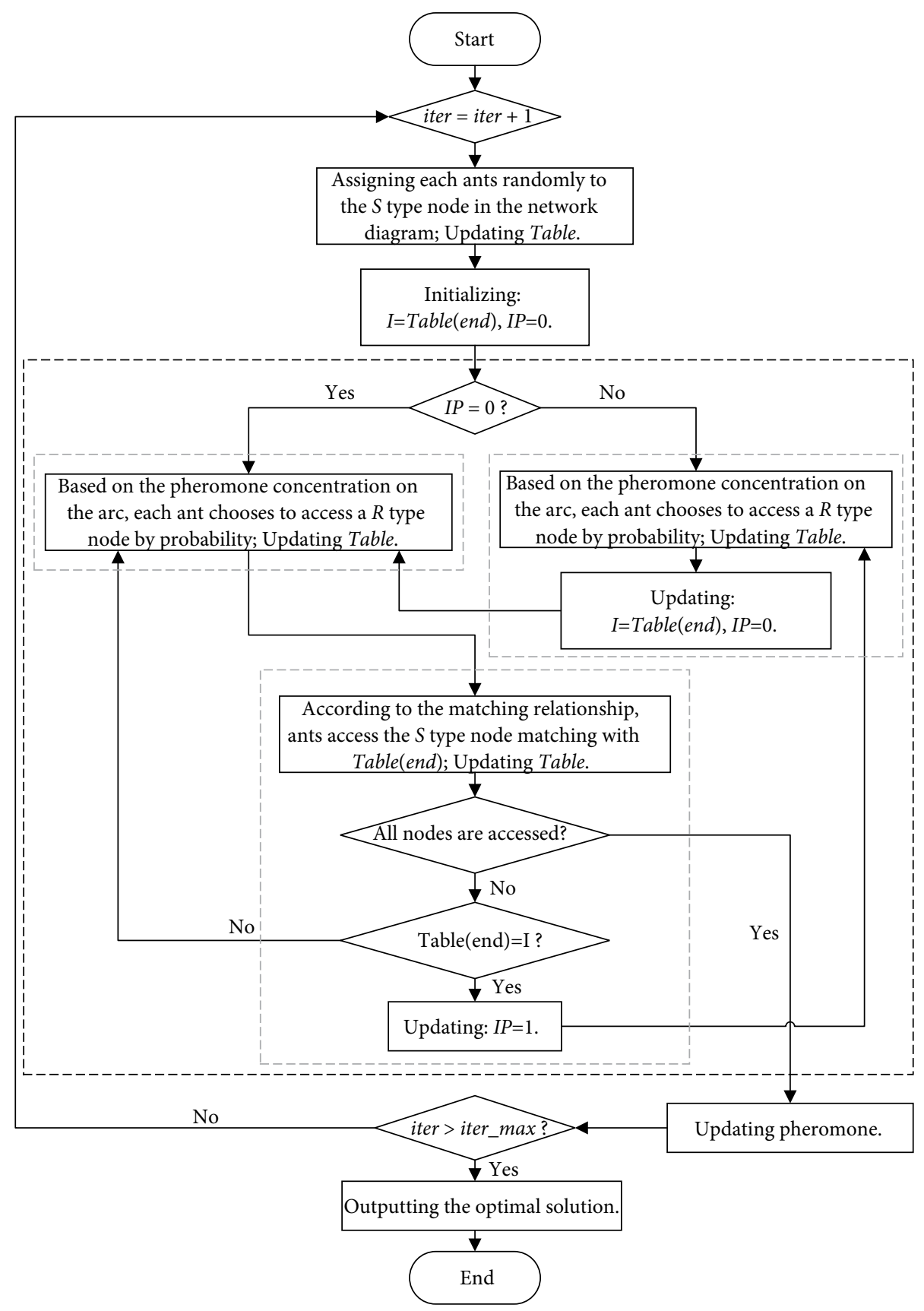

FIgURE 6: The flow chart of ant colony algorithm.

4.4. Converting the Multi-Objective to the SingleObjective. From Section 4.3, the GC-TSP is a multi-objective optimization model. And solution of the multi-objective model is a collection of noninferior solutions. Because each noninferior solution in the collection has the optimal degree, it is difficult for decision makers to make the only optimal decision. Therefore, this paper converts the multi-objective to a single-objective with linear weighting method, and then finds the unique solution under different weights. It should be noted that it is necessary to correct the weights if there is a correlation between the optimization objectives. In addition, since that measurement methods of the operation time and containers unloaded to the ground are different, it is necessary to eliminate the difference of their dimension.

Obviously, $\min T^{\zeta} \geq T^{* \zeta}, \min N^{\zeta} \geq N^{* \zeta}=1$, and then, $0<T^{* \zeta} / \min T^{\zeta} \leq 1,0<N^{* \zeta} / \min N^{\zeta} \leq 1$. And the value of $T^{* \zeta} / \min T^{\zeta}$ and $N^{* \zeta} / \min N^{\zeta}$ are closer to 1 , the better of the result. Therefore, this paper proposes that taking the transshipment efficiency $E_{T N}^{\zeta}$ as the unique indicator measuring the degree of optimization. The calculation of transshipment efficiency $E_{T N}^{\zeta}$ is shown as formula (20), where $0<E<1$. And the transshipment efficiency is higher, if $E_{T N}^{\zeta}$ is closer to 1. 


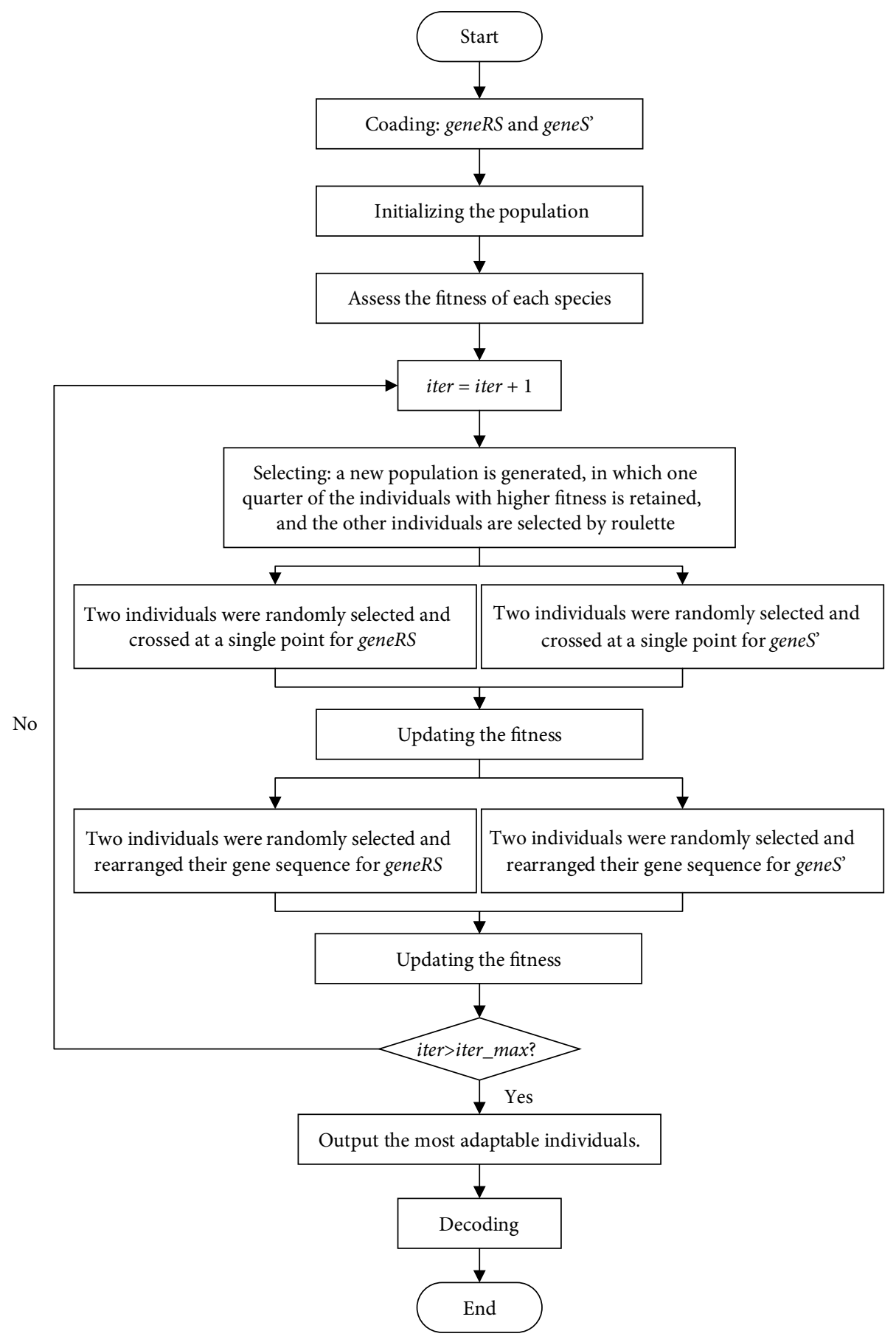

FIgURE 7: The flow chart genetic algorithm.

$$
\begin{aligned}
E_{T N}^{\zeta} & =\max _{N I S^{\zeta}}\left(\alpha \times \frac{T^{* \zeta}}{\min T^{\zeta}}+\beta \times \gamma \times \frac{N^{* \zeta}}{\min N^{\zeta}}\right) \\
& =\max _{N I S^{\zeta}}\left(\alpha \times \frac{T^{* \zeta}}{\min T^{\zeta}}+\beta \times \gamma \times \frac{1}{\min N^{\zeta}}\right) .
\end{aligned}
$$

where, $\alpha$-the coefficient of the weight for "operation time", and $0<\alpha<1, \alpha+\beta=1$.

$\beta$-the coefficient of the weight for the "proportion of containers that are transshipped directly". Here, the meaning of "transshipped directly" is that containers are not being unloaded to the ground. And this paper considers that "the proportion of containers that are transshipped directly" and "the number of containers unloaded to the ground" have the same effect. And $0<\beta<1, \alpha+\beta=1$.

$\gamma$-the correction coefficient of weights, and $0<\gamma<1$.

\section{Algorithm Design}

TSP is a NP-hard problem, it is suitable to use the heuristic algorithm to resolve it. This paper designs the Ant Colony 
TABLE 1: The match table of outbound containers and railway flat cars.

\begin{tabular}{lcccccccccc}
\hline HCs $S_{i}$ & 1 & 2 & 3 & 4 & 5 & 6 & 7 & 8 & 9 & 10 \\
Flat-cars $R_{i}$ & 28 & 31 & 7 & 32 & 18 & 6 & 12 & 17 & 37 & 39 \\
\hline HCs $S_{i}$ & 11 & 12 & 13 & 14 & 15 & 16 & 17 & 18 & 19 & 20 \\
Flat-cars $R_{i}$ & 9 & 40 & 36 & 16 & 27 & 8 & 15 & 33 & 26 & 38 \\
\hline HCs $S_{i}$ & 21 & 22 & 23 & 24 & 25 & 26 & 27 & 28 & 29 & 30 \\
Flat-cars $R_{i}$ & 20 & 3 & 30 & 34 & 21 & 25 & 24 & 14 & 19 & 10 \\
\hline HCs $S_{i}$ & 31 & 32 & 33 & 34 & 35 & 36 & 37 & 38 & 39 & 40 \\
Flat-cars $R_{i}$ & 23 & 1 & 11 & 4 & 5 & 29 & 22 & 13 & 35 & 2 \\
\hline
\end{tabular}

TABLE 2: The basic parameters list.

\begin{tabular}{lccc}
\hline Basic parameters & $v b$ & te & $l$ \\
\hline Unit & $m /$ minute & minute & $m$ \\
Value & 50 & 1.83 & 14 \\
\hline
\end{tabular}

Algorithm and the Genetic Algorithm respectively to resolve the GC-TSP model.

\subsection{The Ant Colony Algorithm}

(1) Symbol Variables

iter-the number of iterations, and initialize it with 0 . iter_max - the maximum of iter, and iter_max $=100$.

$R$-the collection of the node $r_{i}$.

$S$-the collection of the node $s_{j}$.

Table - the record table of path.

Table(end)-the node where ants are located now.

$I$-the latest $s_{j}^{\prime}$ in the path.

$I P$ - the sign triggering the transfer arc, $I P=\{0,1\}$, and the ant reaches next node through the transfer arc if $I P=1$.

(2) Algorithm Flow. The flow of Ant Colony Algorithm is shown in Figure 6.

\subsection{The Genetic Algorithm}

(1) Symbol Variables

iter - the evolutionary algebra.

iter_max - the maximum of iter, and iter_max $=1000$. geneRS - the gene coding, representing the order of the Specified Arcs in the operation sequence.

gene $S^{\prime}$ - the gene coding, representing the order of the Transfer Arcs in the operation sequence.

$n$-the number of individuals in the population, and $n=200$.

(2) Algorithm Flow. The flow of Genetic Algorithm is shown in Figure 7.

\section{Example Analysis}

In order to verify the correctness and effectiveness of the GC-TSP model and its algorithm, this paper takes one working area of the loading and unloading line as the example and studies the optimization of working path of gantry cranes. There is $40 \mathrm{HCs}$ in the working area, and the matching relationship of containers and flat-cars determined by pre-manifest is shown in Table 1.

In the Table 1, numbering HCs according the order of arriving the loading and unloading line, and the railway flatcar are numbered sequentially from one end of the loading and unloading line. Table 2 contains the basic parameter.

6.1. The Optimal Solution by ACA. Resolve the GC-TSP model with ACA, and get non-inferior solutions of the multi-objective optimization, that is the optimal operation time $\min T$ and the optimal number of containers unloaded to the groundmin $N$, as shown in Table 3. And the optimal result of each single-objective are as follow, $T^{*}=281.1077$ minutes, $N^{*}=1$. And the result of nondimensional process are contained in the Table 3 as well.

6.1.1. Calculating the Correction Coefficient. As can be seen in Table 3, the operation time gradually decrease as the number of containers unloaded to the ground increases, which proves the existence of correlation.

Takingmin $T$ and $\min N$ as the independent variable and dependent variable respectively, perform a polynomial fitting, and the curve fitting is shown as Figure 8.

In Figure 8, the pink lines is the tangent of the fitted curve at each discrete point, and the number next to it is the slope, represented by $K$, of each tangent. As can be seen in Figure 8, the absolute value of slop $K$ shows a decreasing trend as the increasing of the number of containers unloaded to the ground, in the other word, the impact of the number of containers unloaded to the ground on the operation time reduces gradually. This paper introduces the Local Impact Factor $f(K)$ to measure the degree that the number of containers unloaded to the ground impacts the operation time. The value range of $f(K)$ is $(0,1]$, and the larger value, the greater impact. In addition, the correlation coefficient $R$ obtained by calculation is -0.8466 , and taking $|R|$ as the Overall Impact Factor. And the calculating of correction coefficients for weights is shown as formula (21).

$$
\gamma=1-\frac{f(K)+|R|}{2} .
$$

The correction coefficient of weights for noninferior solutions is shown in Table 4.

And then, weights that were corrected $\beta \gamma$ are shown in Table 5.

6.1.2. Calculating the Transshipment Efficiency. The transshipment efficiency $E_{T N}$ of noninferior solutions can be obtained by formula (20), as shown in Table 6.

As can be seen in Table 6, the transshipment efficiency $E_{T N}$ decreases gradually as the importance degree of the number of containers unloaded to the ground increases. The optimal solutions under different weights are as follows: if $\alpha \geq 0.8$, the optimal solution is I; the optimal solution is III, if $\alpha<0.8$. In the other word, if the decision maker thinks that the operation time is more important, then I is chosen as the optimal solution. While if the decision maker thinks that the proportion 
TABLE 3: The optimal result by ACA.

\begin{tabular}{lcccccc}
\hline Number & I & II & III & IV & V & VI \\
\hline $\min T$ & 284.8662 & 287.4533 & 287.4544 & 294.4782 & 316.155 & 365.4267 \\
$\min N$ & 7 & 6 & 5 & 4 & 3 & 2 \\
$T^{*} / \min T$ & 0.9868 & 0.9779 & 0.9779 & 0.9546 & 0.8891 & 0.7693 \\
$N^{*} / \min N$ & 0.1429 & 0.1667 & 0.2000 & 0.2500 & 0.3333 & 0.5000 \\
\hline
\end{tabular}

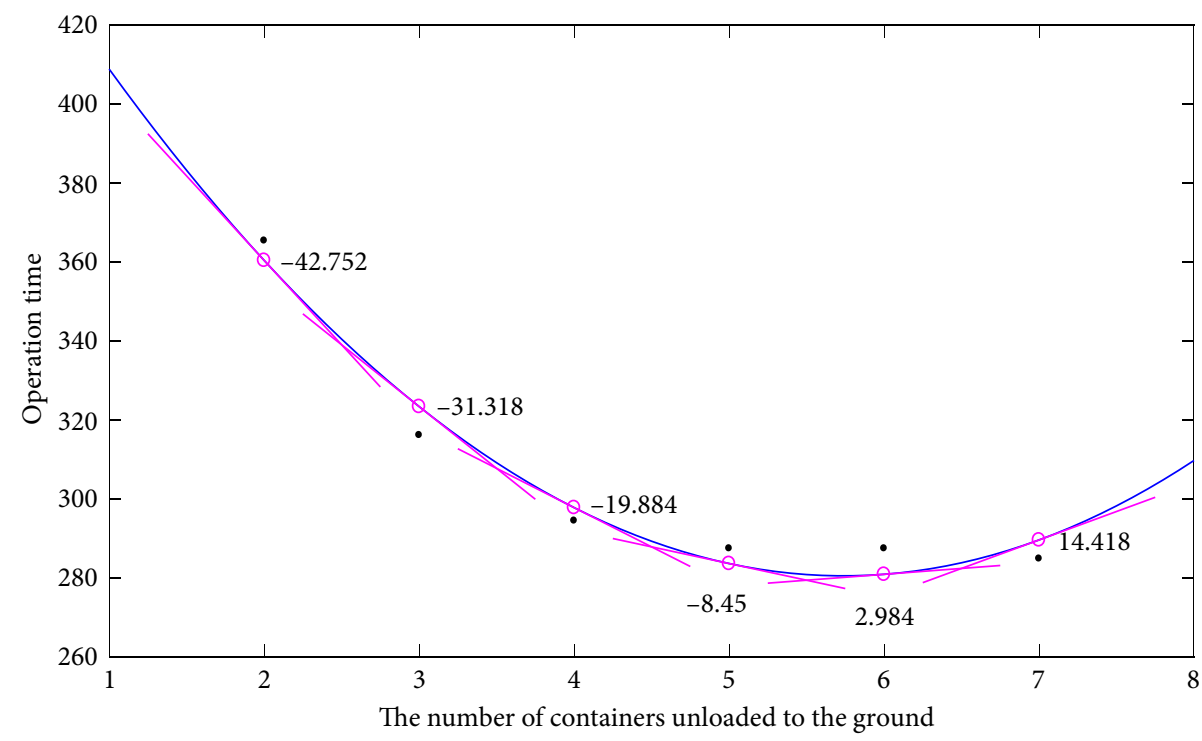

FIGURE 8: The curve of polynomial fitting.

TABLE 4: The correction coefficient of weights.

\begin{tabular}{lcccccc}
\hline $\begin{array}{l}\text { Noninferior } \\
\text { solutions }\end{array}$ & I & II & III & IV & V & VI \\
\hline $\begin{array}{l}\text { Local impact } \\
\text { factors } f(K)\end{array}$ & 0.2 & 0.1 & 0.2 & 0.3 & 0.4 & 0.5 \\
$\begin{array}{l}\text { Overall } \\
\text { impact }\end{array}$ & 0.85 & 0.85 & 0.85 & 0.85 & 0.85 & 0.85 \\
$\begin{array}{l}\text { factors }|R| \\
\begin{array}{l}\text { Correction } \\
\text { coefficients } \gamma\end{array}\end{array}$ & 0.475 & 0.525 & 0.475 & 0.425 & 0.375 & 0.325 \\
\hline
\end{tabular}

TABLE 5: The corrected weight.

\begin{tabular}{cccccccc}
\hline \multicolumn{3}{c}{$\beta \gamma$} & \multicolumn{6}{c}{ Correction coefficients $\gamma$} \\
& & 0.475 & 0.525 & 0.475 & 0.425 & 0.375 & 0.325 \\
\hline \multirow{4}{*}{$\beta$} & 0.1 & 0.0475 & 0.0525 & 0.0475 & 0.0425 & 0.0375 & 0.0325 \\
& 0.2 & 0.0950 & 0.1050 & 0.0950 & 0.0850 & 0.0750 & 0.0650 \\
& 0.3 & 0.1425 & 0.1575 & 0.1425 & 0.1275 & 0.1125 & 0.0975 \\
& 0.4 & 0.1900 & 0.2100 & 0.1900 & 0.1700 & 0.1500 & 0.1300 \\
& 0.5 & 0.2375 & 0.2625 & 0.2375 & 0.2125 & 0.1875 & 0.1625 \\
\hline
\end{tabular}

of containers that are transshipped directly is more important, then III is chosen as the optimal solution.

The working path for solution $I$ is shown in Figure 9. In this figure, the pink line denotes the "specified arc", green line denotes the "random arc", and red line denotes the "transfer arc".
The transshipment sequence for containers in solution I is shown in Table 7.

The working path for solution III is shown in Figure 10.

The transshipment sequence for containers in solution III is shown in Table 8.

From the above, there are 7 containers were unloaded to the ground in solution I, and the proportion of containers that were transshipped directly is $91.25 \%$. There are 5 containers were unloaded to the ground in solution III, and the proportion of containers that were transshipped directly is $93.75 \%$. The total operation time of solution I is 284.87 minutes, and the average operation time per container is 3.56 minutes. The total operation time of solution III is 287.45 minutes, and the average operation time per container is 3.59 minutes.

6.2. The Optimal Solution by GA. Resolve the GC-TSP model with GA, and get non-inferior solutions of the multi-objective optimization, that is the optimal operation time $\min T$ and the optimal number of containers unloaded to the ground $\min N$, as shown in Table 9. And the optimal result of each singleobjective are as follow, $T^{*}=288.2201$ minutes, $N^{*}=1$. And the result of nondimensional process are contained in the as well.

The transshipment efficiency $E_{T N}$ of noninferior solutions can be obtained by formula (20), as shown in Table 10 .

As can be seen in Table 10, the optimal solutions under different weights are as follows: if $\alpha \geq 0.9$, the optimal solution is I; the optimal solution is III, if $\alpha<0.9$. In the other word, if the decision maker thinks that the operation time is more 
TABLE 6: The transshipment efficiency (ETN) for various weights.

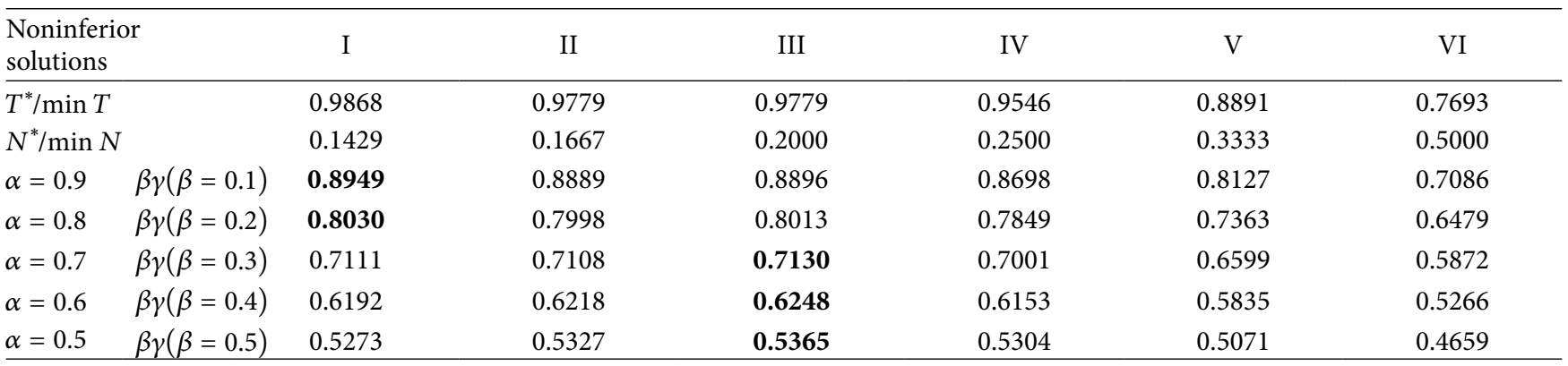

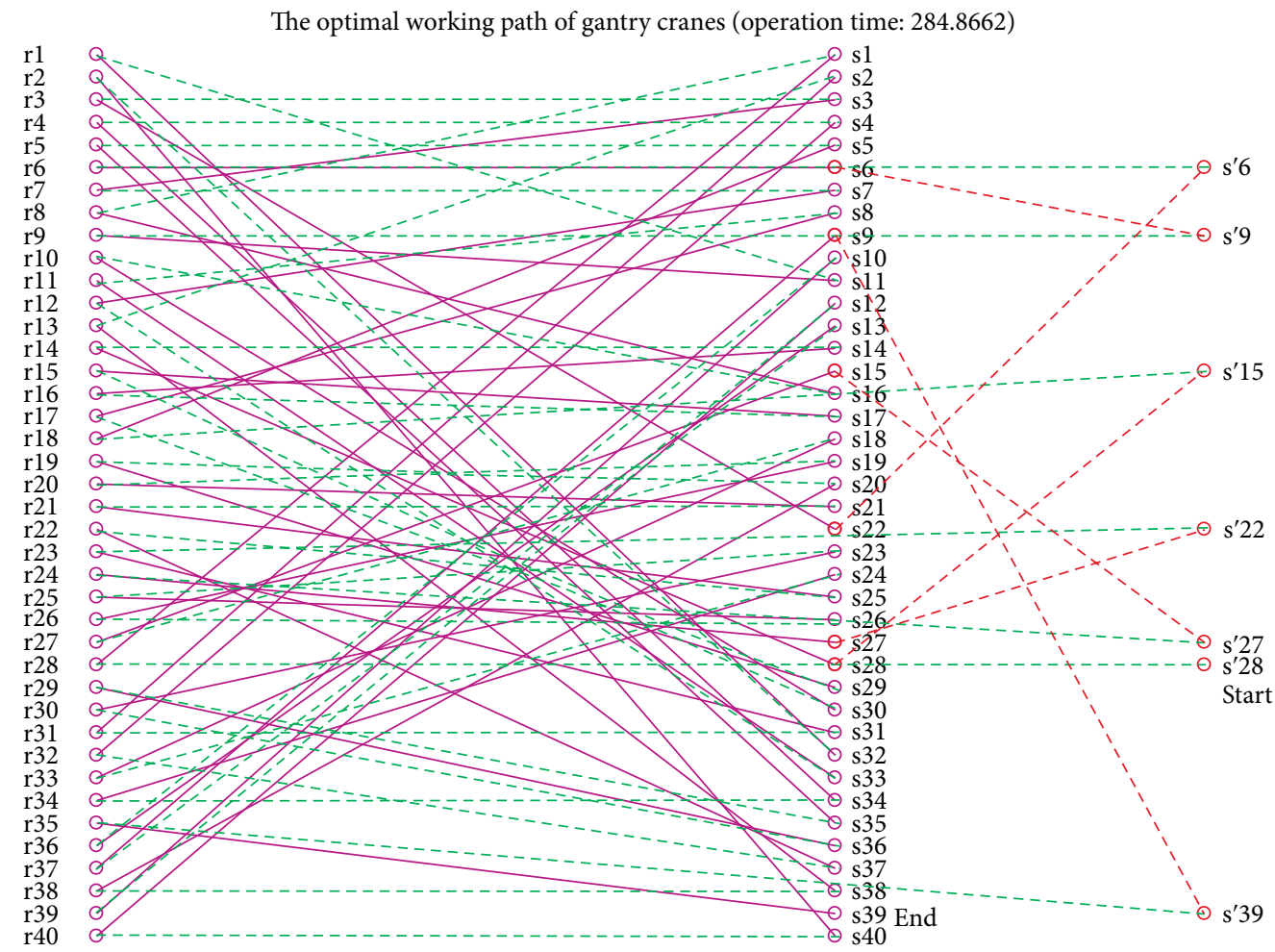

FIgURE 9: The gantry crane's operation path for solution I.

TABLE 7: The transshipment sequence for containers in solution I.

\begin{tabular}{|c|c|c|c|c|c|c|c|c|c|c|c|c|c|c|}
\hline$s^{\prime} 28$ & $r 28$ & $s 1$ & $r 8$ & $s 16$ & $r 10$ & $s 30$ & $r 15$ & $s 17$ & $r 16$ & $s 14$ & $r 14$ & $s 28$ & $s^{\prime} 15$ & $r 18$ \\
\hline$s 5$ & $r 5$ & $s 35$ & $r 29$ & $s 36$ & $r 30$ & $s 23$ & $r 25$ & $s 26$ & $r 26$ & $s 19$ & $r 20$ & $s 21$ & $r 21$ & $s 25$ \\
\hline$r 22$ & $s 37$ & $r 32$ & $s 4$ & $r 4$ & $s 34$ & $r 34$ & $s 24$ & $r 33$ & $s 18$ & $r 27$ & $s 15$ & $s^{\prime} 27$ & $r 24$ & $s 27$ \\
\hline$s^{\prime} 22$ & $r 23$ & $s 31$ & $r 31$ & $s 2$ & $r 13$ & $s 38$ & $r 38$ & $s 20$ & $r 19$ & $s 29$ & $r 17$ & $s 8$ & $r 11$ & $s 33$ \\
\hline$r 12$ & $s 7$ & $r 7$ & $s 3$ & $r 3$ & $s 22$ & $s^{\prime} 6$ & $r 6$ & $s 6$ & $s^{\prime} 9$ & $r 9$ & $s 11$ & $r 1$ & $s 32$ & $r 2$ \\
\hline$s 40$ & $r 40$ & $s 12$ & $r 39$ & $s 10$ & $r 36$ & $s 13$ & $r 37$ & $s 9$ & $s^{\prime} 39$ & $r 35$ & $s 39$ & & & \\
\hline
\end{tabular}

important, then I is chosen as the optimal solution. While if the decision maker thinks that the proportion of containers that are transshipped directly is more important, then III is chosen as the optimal solution.

The total operation time of solution I is 298.3772 minutes, and the average operation time per container is 3.73 minutes. The total operation time of solution III is 301.0012 minutes, and the average operation time per container is 3.76 minutes.

6.3. The Comparison of Results from ACA and GA. Comparing the Tables 6 and 10, the optimal solutions obtained by the two algorithms both are non-inferior solutions I and III, and the proportion of containers that were transshipped directly is the 


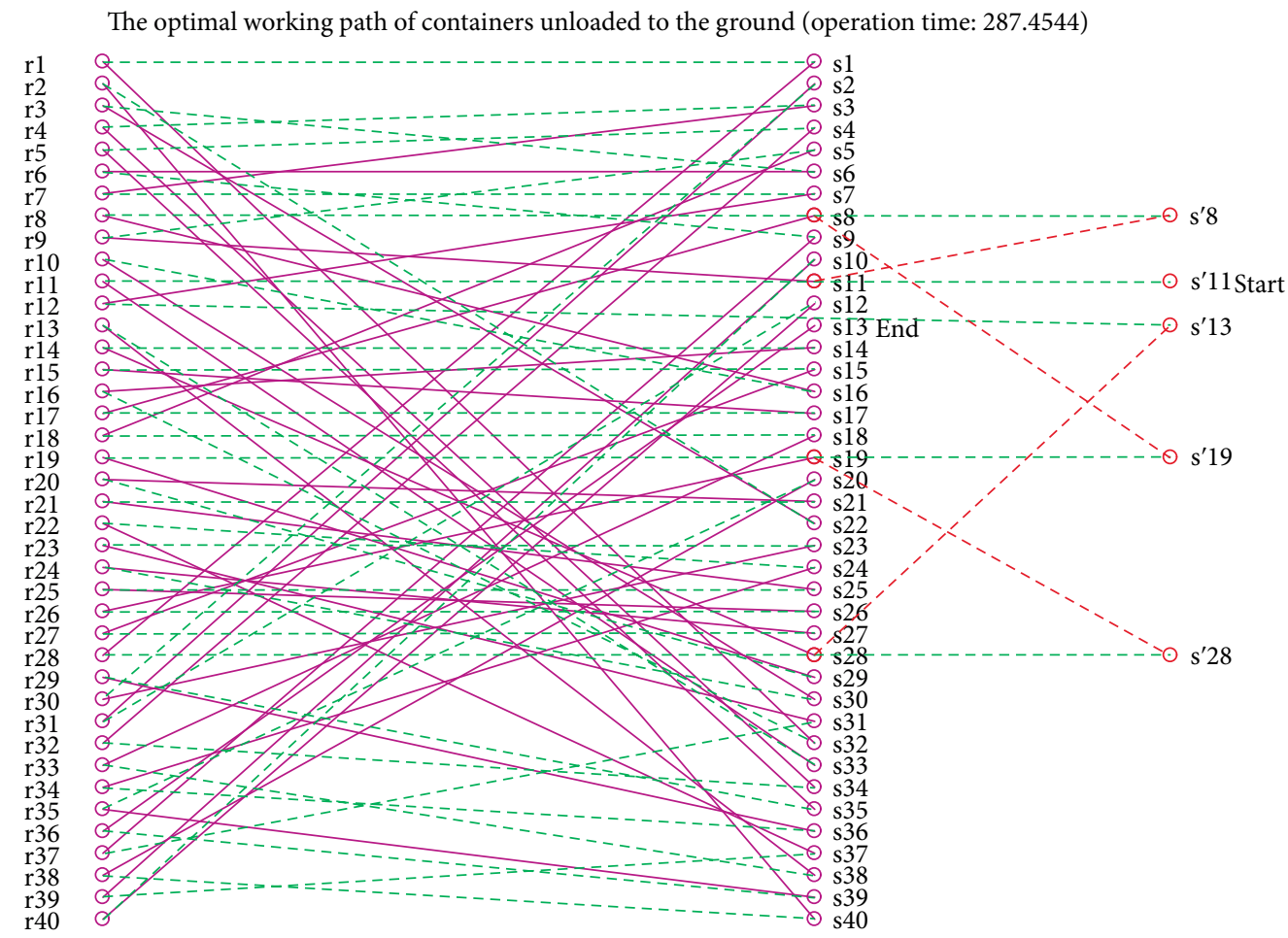

FIGURE 10: The gantry crane's operation path for solution III.

TABLE 8: The transshipment sequence for containers in solution III.

\begin{tabular}{|c|c|c|c|c|c|c|c|c|c|c|c|c|c|c|}
\hline$s^{\prime} 11$ & $r 11$ & $s 33$ & $r 13$ & $s 38$ & $r 33$ & $s 18$ & $r 18$ & $s 5$ & $r 9$ & $s 11$ & $s^{\prime} 8$ & $r 8$ & $s 16$ & $r 10$ \\
\hline$s 30$ & $r 24$ & $s 27$ & $r 27$ & $s 15$ & $r 15$ & $s 17$ & $r 17$ & $s 8$ & $s^{\prime} 19$ & $r 19$ & $s 29$ & $r 20$ & $s 21$ & $r 21$ \\
\hline$s 25$ & $r 25$ & $s 26$ & $r 26$ & $s 19$ & $s^{\prime} 28$ & $r 28$ & $s 1$ & $r 1$ & $s 32$ & $r 16$ & $s 14$ & $r 14$ & $s 28$ & $s^{\prime} 13$ \\
\hline$r 12$ & $s 7$ & $r 7$ & $s 3$ & $r 4$ & $s 34$ & $r 32$ & $s 4$ & $r 5$ & $s 35$ & $r 29$ & $s 36$ & $r 34$ & $s 24$ & $r 22$ \\
\hline$s 37$ & $r 39$ & $s 10$ & $r 40$ & $s 12$ & $r 31$ & $s 2$ & $r 30$ & $s 23$ & $r 23$ & $s 31$ & $r 37$ & $s 9$ & $r 6$ & $s 6$ \\
\hline$r 3$ & $s 22$ & $r 2$ & $s 40$ & $r 38$ & $s 20$ & $r 35$ & $s 39$ & $r 36$ & $s 13$ & & & & & \\
\hline
\end{tabular}

TABLE 9: The optimal result by GA.

\begin{tabular}{lcccccc}
\hline Number & I & II & III & IV & V & VI \\
\hline $\min T$ & 298.3722 & 300.4215 & 301.0012 & 308.2213 & 320.1107 & 369.6613 \\
$\min N$ & 7 & 6 & 5 & 4 & 2 & 0.9351 \\
$T^{*} / \min T$ & 0.9660 & 0.9594 & 0.9575 & 0.9351 & 0.9004 & 0.7797 \\
$N^{*} / \min N$ & 0.1429 & 0.1667 & 0.2 & 0.25 & 0.3333 & 0.5 \\
\hline
\end{tabular}

TABLE 10: The transshipment efficiency $\left(E_{T N}\right)$ for various weights.

\begin{tabular}{|c|c|c|c|c|c|c|}
\hline $\begin{array}{l}\text { Noninferior } \\
\text { solutions }\end{array}$ & I & II & III & IV & $\mathrm{V}$ & VI \\
\hline$T * / \min T$ & 0.9660 & 0.9594 & 0.9575 & 0.9351 & 0.9004 & 0.7797 \\
\hline$N * / \min N$ & 0.1429 & 0.1667 & 0.2 & 0.25 & 0.3333 & 0.5 \\
\hline$\beta \gamma(\beta=0.1)$ & 0.8613 & 0.8527 & 0.8513 & 0.8311 & 0.8196 & 0.6989 \\
\hline$\beta \gamma(\beta=0.2)$ & 0.8001 & 0.8019 & 0.8100 & 0.7708 & 0.7304 & 0.6660 \\
\hline$\beta \gamma(\beta=0.3)$ & 0.6991 & 0.7010 & 0.7061 & 0.6988 & 0.6410 & 0.5714 \\
\hline$\beta \gamma(\beta=0.4)$ & 0.6013 & 0.6193 & 0.6214 & 0.6019 & 0.5471 & 0.5180 \\
\hline$\beta \gamma(\beta=0.5)$ & 0.5207 & 0.5211 & 0.5219 & 0.5177 & 0.4559 & 0.4106 \\
\hline
\end{tabular}


same. The difference is: (1) if $\alpha$ takes 0.8 , the optimal solution obtained by ACA is I, while the optimal solution obtained by GA is III; (2) the transshipment efficiency for ACA is higher than GA; (3) for the average operation time per container, the result of ACA is 3.6 minutes, while the result of GA is 3.7 minutes.

The average operation time per container from ACA and GA are basically consistent, which verifies the correctness and effectiveness of the GC-TSP model and its algorithm. And ACA is superior to GA.

\section{Conclusion}

This paper proposes a method of optimizing the working path of gantry cranes for the problem that containers are transshipped directly under the matching relationship. This paper converts the optimization problem to a TSP problem, constructs the GC-TSP model. And then, the paper designs ACA and GA respectively to solve the GC-TSP model.

The result shows that the proportion of containers that were transshipped directly is 90 percent or more. And the average operation time per container calculated by the two algorithms are basically consistent, which verifies the correctness and effectiveness of the GC-TSP model and its algorithms.

\section{Data Availability}

Major part of the data used to support the findings of this study are included within the article. Further information can be obtained from author Mr. Dong.

\section{Conflicts of Interest}

The authors declare that they have no conflicts of interest.

\section{Acknowledgments}

These positions are funded by 2016 China's National Key Research and Strategic International Science and Technology Innovation Cooperation Project, which is titled "'the Belt and Road' Land Transport International Intermodal Research and Exchange Centre" under Grant [2016YFE0201700].

\section{References}

[1] S. Y. Zhu, Handling Scheduling Optimization for Railway Container Terminals based on Train Arrival-Departure Time, Beijing Jiaotong University, Beijing, 2016.

[2] S. X. Xu, Scheduling Optimization of Loading/Unloading Transfer Operations Considering Rehandling Activities in Railway Container Terminals, Southwest Jiaotong University, Chengdu, 2018.

[3] D. Briskorn, S. Emdeb, and N. Boysenb, "Cooperative twincrane scheduling," Discrete Applied Mathematics, vol. 2016, no. 211 , pp. $40-57,2016$.
[4] F. Jaehn and D. Kress, "Scheduling cooperative gantry cranes with seaside and landside jobs," Discrete Applied Mathematics, vol. 2018, no. 242, pp. 53-68, 2018.

[5] L. L. Chen, "Research on container yard crane routing problem based on state node network optimization," Operations Research and Management Science, vol. 25, no. 5, pp. 82-87, 2016.

[6] X. Y. Li, A. Otto, and E. Pesch, "Solving the single crane scheduling problem at rail transshipment yards," Discrete Applied Mathematics, vol. 2019, no. 264, pp. 134-147, 2019.

[7] S. Heshmatia, T. A. M. Toffoloa, and W. Vancroonenburga, "Crane-operated warehouses: integrating location assignment and crane scheduling," Computers \& Industrial Engineering, vol. 2019, no. 129, pp. 274-295, 2019.

[8] N. Al-Dhaheri, A. Jebali, and A. Diabat, "A simulation-based Genetic Algorithm approach for the quay crane scheduling under uncertainty," Simulation Modelling Practice and Theory, vol. 66, pp. 122-138, 2016.

[9] N. Al-Dhaheri and A. Diabat, "The quay crane scheduling problem," Journal of Manufacturing Systems, vol. 36, pp. 87-94, 2015.

[10] D. Kress, J. Dornseifer, and F. Jaehn, "An exact solution approach for scheduling cooperative gantry cranes," European Journal of Operational Research, vol. 2019, no. 273, pp. 82-101, 2019.

[11] M. Böck, A. Stöger, and A. Kugi, "Efficient generation of fast trajectories for gantry cranes with constraints," IFACPapersOnLine, vol. 50, no. 1, pp. 1937-1943, 2017.

[12] E. Benavent, M. Landete, J-J. Salazar-González, and G. Tirado, "The probabilistic pickup-and-delivery travelling salesman problem," Expert Systems with Applications, vol. 121, pp. 313323, 2019.

[13] P. A. Miranda, C. A. Blazquez, C. Obreque, J. Maturana-Ross, and G. Gutierrez-Jarpa, "The bi-objective insular traveling salesman problem with maritime and ground transportation costs," European Journal of Operational Research, vol. 271, no. 3, pp. 1014-1036, 2018.

[14] J. W. Li, Research on TSP Problem of Logistics Distribution, Harbin Institute of Technology, Harbin, 2017.

[15] H. J. Dong, Optimization of Gantry Cranes' Operation Path for Transshipment based on Improved TSP, Beijing Jiaotong University, Beijing, 2019. 


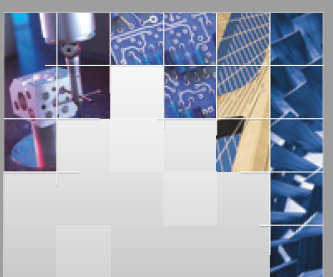

\section{Enfincering}
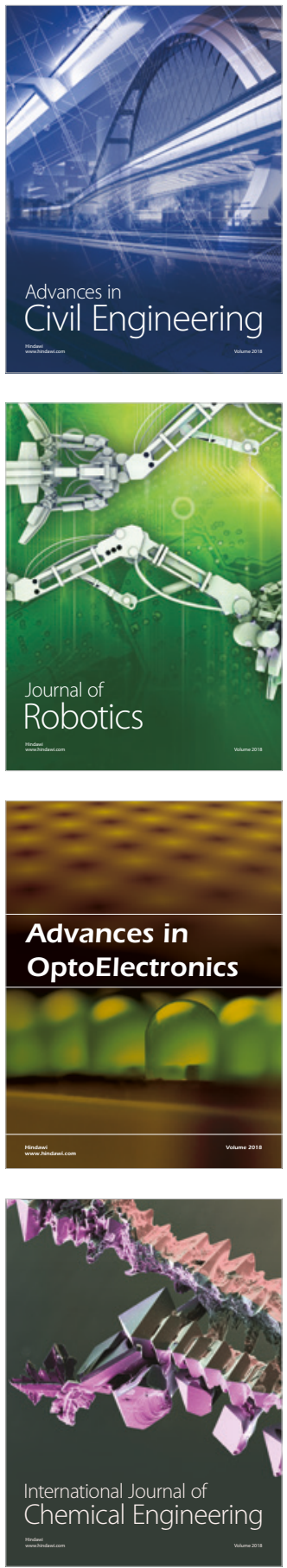

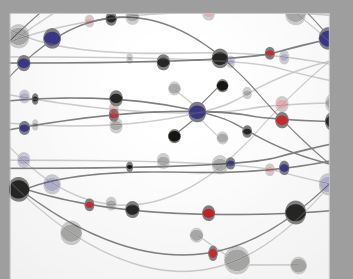

\section{Rotating \\ Machinery}

The Scientific World Journal

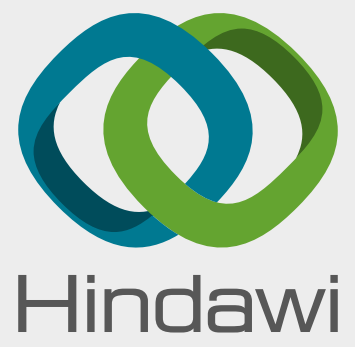

Submit your manuscripts at

www.hindawi.com
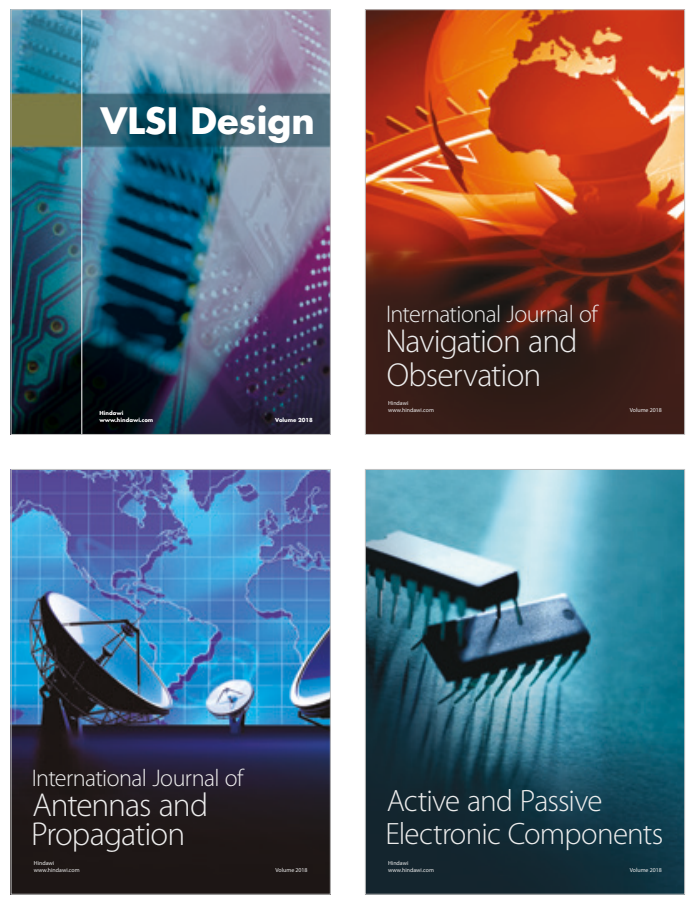
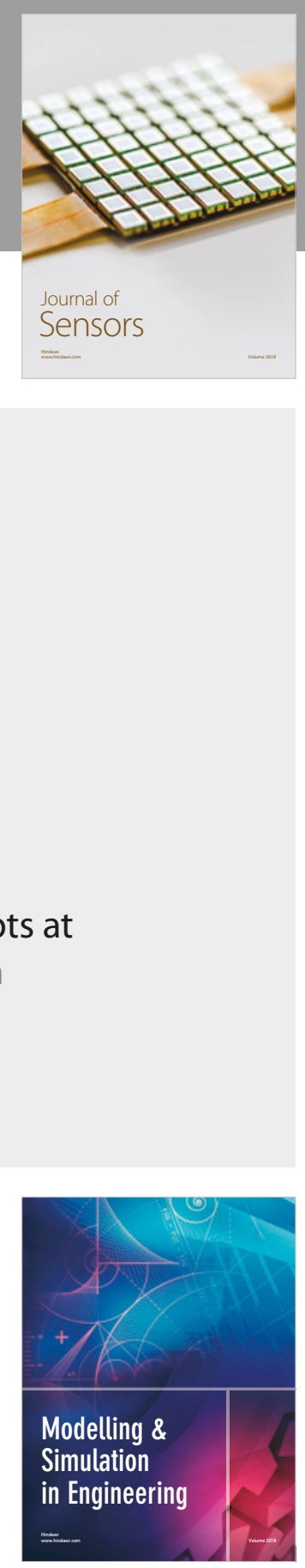

\section{Advances \\ Multimedia}
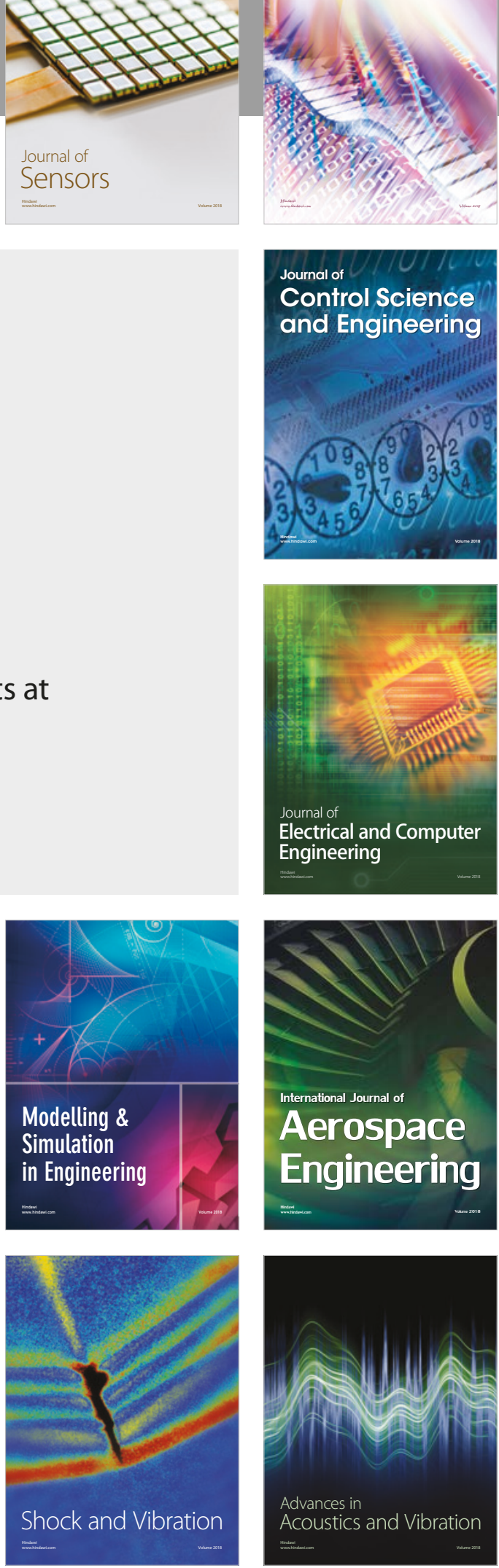